\title{
Erratum to: High Jagged1 expression is associated with poor outcome in primary glioblastoma
}

\author{
Xian-xin Qiu ${ }^{1} \cdot$ Chen-hong Wang ${ }^{2} \cdot \mathrm{Na} \mathrm{You}^{2} \cdot$ Bi-juan Chen ${ }^{2} \cdot$ Xing-fu Wang ${ }^{3} \cdot$ \\ Yu-peng Chen ${ }^{3} \cdot$ Zhi-xiong Lin ${ }^{1,2}$
}

Published online: 27 July 2016

(C) Springer Science+Business Media New York 2016

\section{Erratum to: Med Oncol (2015) 32:341 \\ DOI 10.1007/s12032-014-0341-9}

The original version of this article unfortunately contained a mistake. The authors found the original Figure 1 was incorrect as described and not created for this article. Authors respectfully correct their Fig. 1 and legend through this erratum.

The online version of the original article can be found under doi:10.1007/s12032-014-0341-9.

Zhi-xiong Lin

1zx1967@sina.com

1 Department of Neurosurgery, The First Affiliated Hospital, Fujian Medical University, Fuzhou 350005, Fujian, China

2 Tumor Invasion Microecological Laboratory, Fujian Medical University, Fuzhou, Fujian, China

3 Department of Pathology, The First Affiliated Hospital, Fujian Medical University, Fuzhou, Fujian, China 

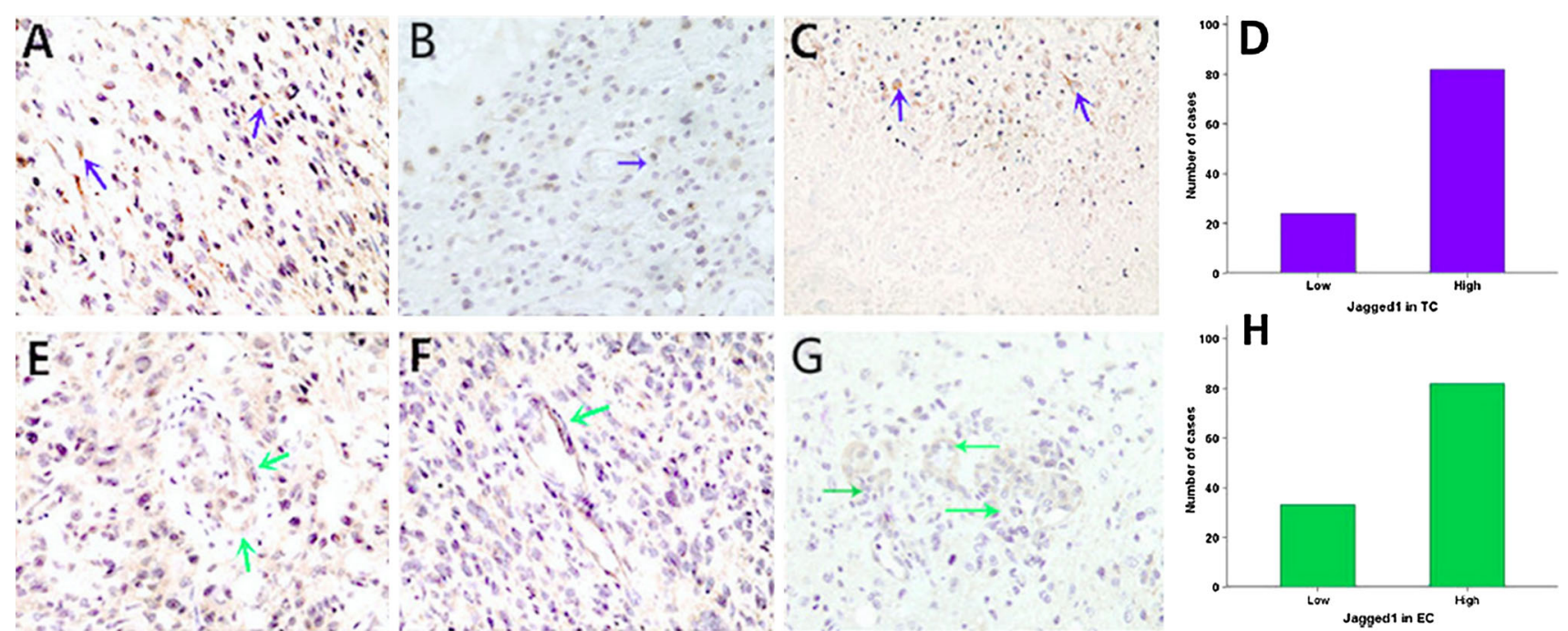

Fig. 1 Degree and cellular distribution of Jagged1 expression in glioblastoma tissues. a-d Jagged1-positive cells in tumor cells (blue arrow), seen in tumor cells with diffuse distribution (a), perivascular distribution (b) or adjacent necrosis (c). e-h Jagged1 expression in

endothelial cells (green arrow), including endothelial cells of glomeruloid vessels (e), scattered non-glomeruloid vessels (h) and clustered non-glomeruloid vessels $(\mathbf{g})$ 